\title{
Photonic Gravitational Interactions from a Quantum Point of View
}

\author{
Jose L. Parra \\ Physics Department, Florida International University, Miami, USA \\ Email: JLparra@fiu.edu
}

How to cite this paper: Parra, J.L. (2021) Photonic Gravitational Interactions from a Quantum Point of View. Optics and Photonics Journal, 11, 12-21. https://doi.org/10.4236/opj.2021.111002

Received: December 10, 2020

Accepted: January 26, 2021

Published: January 29, 2021

Copyright (C) 2021 by author(s) and Scientific Research Publishing Inc. This work is licensed under the Creative Commons Attribution International License (CC BY 4.0).

http://creativecommons.org/licenses/by/4.0/

\begin{abstract}
A hypothesis explaining the diffraction and interference of light from a pure corpuscular point of view was published in 2018. The author developed the idea by a fortunate combination of intuition and statistics but failed to justify it theoretically. This vagueness can be amended by using relativistic invariants. Adapting Dirac's equation to gravitational potentials acting over photons yields most of the properties of light. A complete characterization of the properties of light arriving from distant galaxies was performed by modeling the coherence of light. It was assumed that the coherence of light is generated by two orthogonal potentials. Here an idea explains the cosmological redshift data as is done by the combination of Big-Bang, acceleration, and deceleration trilogy.
\end{abstract}

\section{Keywords}

Hubble's Constant, Relativistic Invariants, Dirac's Equation, Cosmology, Redshift

\section{Introduction}

The discovery of a universe populated by many galaxies soon created a new problem within Astronomy. Hubble [1] documented in 1924 a linear relationship between the redshifted light emitted by those galaxies and the distance between them. A natural hypothesis from that observation is that the energy of photons weakens proportionally to the distance traveled. This is a simple idea that does not work well because it fails to explain other experimental results. An unusual hypothesis is to assume that all galaxies are moving away from each other with a velocity proportional to the distance between every pair. Doppler's effect [2] works so well that modern astronomical literature is now showing graphs illustrating the shift of light with velocity. However, it excludes the dis- 
tance which is the real experimental factor. An additional stronger uncommon hypothesis [3] that holds even better is the assumption that the space between galaxies is expanding. The beam expansion of light coming from distant galaxies and the increase in wavelength correlate well with the expansion rate of space. An advantage of that model is that farther galaxies can even retreat at a greater "speed" than the speed of light because space does not carry information. The limit velocity imposed by the special theory of relativity (STR) of Einstein [4] is not violated by this theory. The discovery of the cosmic microwave background radiation $(\mathrm{CMB})$ in $[5]$ increases the complexity of the understanding of cosmology because any model should explain the redshift and the origin of the CMB. A problem with Doppler's effect and space expansion theory is that it is required two independent ideas to explain the variation in light properties. However, light is the carrier of the redshifted effect and the CMB. In other words, the three explanations are not part of a unique independent self-consistent model.

Let us visualize this galactic problem as part of another more universal, simple, and general problem in space and time. Here, a minimum number of postulates having a general fashion are used. Older well-established postulates are reintroduced with the necessary variation to make them universal. The calculations presented later will impose the necessary clarifications to reconnect this idea with the observed data.

\section{Model}

Assume the following:

1) The light coherence state is a set of particles interacting in multiple directions [6].

2) A speed limit $(\Lambda)$ exists in space. A consequence of the STR is that the speed of light $(c)$ is that limit. In general, this does not have to be the case.

3) The Dirac's equation [7], which is relativistically invariant, could be extended to a general expression. Its formalism could be applied to any potential. These potentials could be electrically acting on charges $e$ or could be gravitationally acting on rest masses $\mathrm{m}$.

Dirac's equation for particles that are not complex, as electrons, has the form,

$$
i \hbar \frac{\partial \psi}{\partial t}=\left[c \boldsymbol{\alpha} \cdot(\boldsymbol{p}-e \boldsymbol{A})-e \phi+\beta m c^{2}\right] \psi,
$$

where $I^{2}=-1, \hbar$ is the Planck's constant divided by $2 \pi, \psi$ is the state function, $t$ is time, the vector $\alpha$ is a four-by-four matrix containing the Pauli's matrices, vector $p$ is the four momentum, scalar $\phi$ and vector $A$ are the electrical potentials, $\beta$ is another four-by-four matrix fill up with positive and negative ones, and zeros. Equation (1) is rewritten according to the previous postulates by introducing two variations. The invariant particle charge $e$ is substituted with the rest mass $m$, which is another invariant particle scalar. This implies that in Equation (2), the scalar and vector potentials are now gravitational.

$$
i \hbar \frac{\partial \psi}{\partial t}=\left[\Lambda \boldsymbol{\alpha} \cdot(\boldsymbol{p}-m \boldsymbol{A})-m \phi+\beta m \Lambda^{2}\right] \psi .
$$


Photons with increasing coherence while traveling through space must create a pattern around a line that will be used as the cylindrical coordinate $z$-axis. The state $\psi$ could be represented with a matrix of four components $u_{i}$, where $i$ ranges from one to four, as in [8]. Equation (2) can be split, after some matrix calculation and transformations to cylindrical coordinates, into four interdependent equations, as shown in Equation (3):

$$
\begin{aligned}
& \frac{\partial u_{1}}{\Lambda \partial t}=\frac{i}{\hbar \Lambda}\left[E+m\left(\varphi_{\rho, \phi, z}-\Lambda^{2}\right)\right] u_{1}-\mathrm{e}^{-i \phi} \frac{\partial u_{4}}{\partial \rho}+\frac{i}{\rho} \mathrm{e}^{-i \phi} \frac{\partial u_{4}}{\partial \phi}-\frac{\partial u_{3}}{\partial z} \\
& \frac{\partial u_{2}}{\Lambda \partial t}=\frac{i}{\hbar \Lambda}\left[E+m\left(\varphi_{\rho, \phi, z}-\Lambda^{2}\right)\right] u_{2}-\mathrm{e}^{i \phi} \frac{\partial u_{3}}{\partial \rho}-\frac{i}{\rho} \mathrm{e}^{i \phi} \frac{\partial u_{3}}{\partial \phi}+\frac{\partial u_{4}}{\partial z} \\
& \frac{\partial u_{3}}{\Lambda \partial t}=\frac{i}{\hbar \Lambda}\left[E+m\left(\varphi_{\rho, \phi, z}+\Lambda^{2}\right)\right] u_{3}+\mathrm{e}^{i \phi} \frac{\partial u_{2}}{\partial \rho}+\frac{i}{\rho} \mathrm{e}^{-i \phi} \frac{\partial u_{2}}{\partial \phi}-\frac{\partial u_{1}}{\partial z} \\
& \frac{\partial u_{4}}{\Lambda \partial t}=\frac{i}{\hbar \Lambda}\left[E+m\left(\varphi_{\rho, \phi, z}+\Lambda^{2}\right)\right] u_{4}-\mathrm{e}^{i \phi} \frac{\partial u_{1}}{\partial \rho}-\frac{i}{\rho} \mathrm{e}^{i \phi} \frac{\partial u_{1}}{\partial \phi}+\frac{\partial u_{2}}{\partial z}
\end{aligned}
$$

The symmetrical photon distribution allows the substitution of the functions $u_{i}$ with the product of the four independent functions associated with the corresponding coordinate variables, i.e., $u=R(\rho) \Phi(\phi) Z(z) T(t)$. Set (3) simplifies to set (4) after assuming that $z$ is the direction of travel $(z=c t)$ and a constant time $t$ and a constant radius $\rho$ :

$$
\begin{aligned}
& 0=\frac{\rho}{\hbar \Lambda}\left[E+m\left(\varphi_{\rho, \phi, Z}-\Lambda^{2}\right)\right] R_{1} \Phi_{1} Z_{1} \mathrm{e}^{i \phi}+R_{4} Z_{4} \frac{\partial \Phi_{4}}{\partial \phi} \\
& 0=\frac{\rho}{\hbar \Lambda}\left[E+m\left(\varphi_{\rho, \phi, Z}-\Lambda^{2}\right)\right] R_{2} \Phi_{2} Z_{2} \mathrm{e}^{-i \phi}-R_{3} Z_{3} \frac{\partial \Phi_{3}}{\partial \phi} \\
& 0=\frac{\rho}{\hbar \Lambda}\left[E+m\left(\varphi_{\rho, \phi, Z}+\Lambda^{2}\right)\right] R_{3} \Phi_{3} Z_{3} \mathrm{e}^{i \phi}+R_{2} Z_{2} \frac{\partial \Phi_{2}}{\partial \phi} \\
& 0=\frac{\rho}{\hbar \Lambda}\left[E+m\left(\varphi_{\rho, \phi, Z}+\Lambda^{2}\right)\right] R_{4} \Phi_{4} Z_{4} \mathrm{e}^{-i \phi}-R_{1} Z_{1} \frac{\partial \Phi_{1}}{\partial \phi}
\end{aligned}
$$

The derivation of the top equation of set (4) with respect to the angle $\phi$ can be placed at the bottom equation resulting in a second-grade differential equation for the angular function 4. Angular function 3 can be found in the same way. Angular functions 1 and 2 come directly from the first two equations. It is assumed that the product mass times the potential is very small in comparison with the energy. The solution of set (4) imposes the constriction

$$
l^{2}=\left(\frac{\rho}{\hbar \Lambda}\right)^{2}\left[\left(E+m \varphi_{\rho, \phi, z}\right)^{2}-m^{2} \Lambda^{4}\right]
$$

where $l$ is an integer. As the rest mass $m$ approaches zero, the constriction reduces to

$$
l \approx \frac{2 \pi \rho}{\lambda} \frac{c}{\Lambda},
$$

where $\lambda$ behave as a repetition-length. The last simplified result brings the first support of this idea because the study of the interference of light coming from a far-off star results in the same circular pattern. For example, in the visible region, 
violet is the color of the first circle, followed by indigo and so on. The solutions to Set (4) are the angular Function (5):

$$
\begin{aligned}
& \Phi_{1}=-i \frac{\frac{2 \rho R_{4} Z_{4} T_{4}}{\hbar \Lambda R_{1} Z_{1} T_{1}}\left[E+m\left(\varphi_{\rho, \phi, Z}+\Lambda^{2}\right)\right]}{\sqrt{1+4 l^{2}}-1} \Phi_{0} \mathrm{e}^{\frac{i}{2}\left(\sqrt{1+4 l^{2}}-1\right) \phi} \\
& \Phi_{2}=-i \frac{\frac{2 \rho R_{3} Z_{3} T_{3}}{\hbar \Lambda R_{2} Z_{2} T_{2}}\left[E+m\left(\varphi_{\rho, \phi, Z}+\Lambda^{2}\right)\right]}{\sqrt{1+4 l^{2}}-1} \Phi_{0} \mathrm{e}^{-\frac{i}{2}\left(\sqrt{1+4 l^{2}}-1\right) \phi} \\
& \Phi_{3}=\Phi_{0} \mathrm{e}^{-\frac{i}{2}\left(\sqrt{1+4 l^{2}}+1\right) \phi} \\
& \Phi_{4}=\Phi_{0} \mathrm{e}^{\frac{i}{2}\left(\sqrt{1+4 l^{2}}+1\right) \phi}
\end{aligned}
$$

where numerators in Functions 1 and 2 are integers. Angular Functions 1 and 2 together with Functions 3 and 4 offer the second support of this calculus, which is light showing both clockwise and counterclockwise behavior. These Functions 1 and 2 also exhibit some peculiarities. An integer $l$ with a null value implies a photon with zero momentum, no angular variations, and infinite amplitude. The meaning of that infinitude remains an open question. It is known that the $\mathrm{Di}$ rac's equation introduced the concept of matter and antimatter. Functions 3 and 4 in set (5) are completely fine with matter and antimatter photons at rest. Equations (8) define the status of matter-photons and antimatter-photons.

The four radial functions $R(\rho)$ can be found by returning to Set (3), with $\rho$ being the only variable. The math simplifies because by using the angular Functions (5), the four differential equations are now of the first grade. Their solutions are:

$$
\begin{aligned}
& R_{1}=R_{0}\left(\frac{\rho}{\rho_{0}}\right)^{-\frac{1}{2}\left(\sqrt{1+4 l^{2}}-1\right)} \\
& R_{2}=R_{0}\left(\frac{\rho}{\rho_{0}}\right)^{-\frac{1}{2}\left(\sqrt{1+4 l^{2}}+1\right)} \\
& R_{3}=R_{0} \exp \left\{-\frac{\left[\left(E+m \varphi_{\rho, \phi, Z}\right)^{2}-m^{2} \Lambda^{4}\right]\left(\rho-\rho_{0}\right)^{2}}{(\hbar \Lambda)^{2}\left(1+\sqrt{1+4 l^{2}}\right)}\right\} \\
& R_{4}=R_{0} \exp \left\{-\frac{\left[\left(E+m \varphi_{\rho, \phi, Z}\right)^{2}-m^{2} \Lambda^{4}\right]\left(\rho-\rho_{0}\right)^{2}}{(\hbar \Lambda)^{2}\left(1+\sqrt{1+4 l^{2}}\right)}\right\}
\end{aligned}
$$

The traveling functions arise from making the $z$ direction the only variable and applying the same technique as used before over the angular functions. The solutions this time are identical:

$$
Z_{1}=Z_{2}=Z_{3}=Z_{4}=Z_{0} \mathrm{e}^{\frac{i}{\hbar \Lambda} \sqrt{\left(E+m \varphi_{\rho, \phi, z}\right)^{2}-m^{2} \Lambda^{4} z}} .
$$

The exponential part of the functions $Z$ can be interpreted physically in this way: The real part is associated with gravitational potentials on the line of prop- 
agation. The imaginary part is associated with gravitational potentials perpendicular to the line of propagation. This result becomes the physical-mathematical justification of the idea used in [6]. It is the existence of two independent potentials on (7) doubles the number of states in a sampling of photons. Thus, it is unnecessary to assume two states of polarization, as was desired by Bose [9] who needed that duplication to correlate his statistical approach with the one carried out by Planck [10] in the study of black body radiation.

Working with $t$ as the only local variable yields Solution (8). This solution differentiates, at least mathematically, matter photons from antimatter photons. Equation (8) contains periodic oscillations in time that can be visualized as photons arriving to the observation point according to some repetition rate.

$$
T_{1}=T_{2}=T_{0} \mathrm{e}^{\frac{i}{\hat{\hbar}\left[E+m\left(\varphi-\Lambda^{2}\right)\right] t}}, \quad T_{3}=T_{4}=T_{0} \mathrm{e}^{\frac{i}{\hbar}\left[E+m\left(\varphi+\Lambda^{2}\right)\right] t}
$$

The final solutions come from the combination of Sets (5-7). Solutions for $T_{i}$ are not included here due to a lack of space.

$$
\begin{aligned}
u_{1}= & -i R_{0} \Phi_{0} Z_{0} T_{1} \frac{2 \rho\left[E+m\left(\varphi+\Lambda^{2}\right)\right]}{\hbar \Lambda\left(\sqrt{1+4 l^{2}}-1\right)} \exp \left[\frac{i}{\hbar \Lambda}\left(\sqrt{\left(E+m \varphi_{\rho, \phi, z}\right)^{2}-m^{2} \Lambda^{4}}\right) z\right. \\
& \left.-\frac{\left(E+m \varphi_{\rho, \phi, z}\right)^{2}-m^{2} \Lambda^{4}}{(\hbar \Lambda)^{2}\left(1+\sqrt{1+4 l^{2}}\right)}\left(\rho-\rho_{0}\right)^{2}-\frac{i}{2}\left(1-\sqrt{1+4 l^{2}}\right) \phi\right] \\
u_{2}= & -i R_{0} \Phi_{0} Z_{0} T_{2} \frac{2 \rho\left[E+m\left(\varphi+\Lambda^{2}\right)\right]}{\hbar \Lambda\left(\sqrt{1+4 l^{2}}-1\right)} \exp \left[\frac{i}{\hbar \Lambda}\left(\sqrt{\left(E+m \varphi_{\rho, \phi, z}\right)^{2}-m^{2} \Lambda^{4}}\right) z\right. \\
& \left.-\frac{\left(E+m \varphi_{\rho, \phi, z}\right)^{2}-m^{2} \Lambda^{4}}{(\hbar \Lambda)^{2}\left(1+\sqrt{1+4 l^{2}}\right)}\left(\rho-\rho_{0}\right)^{2}+\frac{i}{2}\left(1-\sqrt{1+4 l^{2}}\right) \phi\right] \\
u_{3}= & R_{0} \Phi_{0} Z_{0} T_{3} \exp \left[\frac{i}{\hbar \Lambda}\left(\sqrt{\left(E+m \varphi_{\rho, \phi, z}\right)^{2}-m^{2} \Lambda^{4}}\right) z\right. \\
& -\frac{(E+m \varphi)^{2}-m^{2} \Lambda^{4}}{(\hbar \Lambda)^{2}\left(1+\sqrt{1+4 l^{2}}\right)}\left(\rho-\rho_{0}\right)^{2}-\frac{i}{2}\left(1+\sqrt{1+4 l^{2}}\right) \phi \\
& \left.-\frac{(E+m \varphi)^{2}-m^{2} \Lambda^{4}}{(\hbar \Lambda)^{2}\left(1+\sqrt{1+4 l^{2}}\right)}\left(\rho-\rho_{0}\right)^{2}+\frac{i}{2}\left(1+\sqrt{1+4 l^{2}}\right) \phi\right] \\
u_{4}= & R_{0} \Phi_{0} Z_{0} T_{4} \exp \left[\frac{i}{\hbar \Lambda}\left(\sqrt{\left(E+m \varphi_{\rho, \phi, z}\right)^{2}-m^{2} \Lambda^{4}}\right) z\right.
\end{aligned}
$$

The energy of light, in principle, can suffer variations over time during long travels, as is the case for intergalactic travels. The calculation of the energy variation with time is a long mathematical process that requires some clarification 
and simplification. The spatial variable $\rho$ remains independent of time only if the variations in the energy occur in jumps. The reason for this is the constriction created by the solution to (4) that connects the energy $E$ with the integer 1 . Let us assume that the rest mass squared goes to zero. Then, the constriction becomes

$$
l \approx \frac{\rho}{\hbar \Lambda}\left(E+m \varphi_{\rho, \phi, z}\right)
$$

and Set (9), after remembering that $z=c t$, is simplified to Set (10) if $l \gg 1$ is also assumed. This condition fails for extremely large trips were light is highly redshifted. The corresponding math for very large trips will be introduced in another paper to study the origin of (CMB) radiation.

$$
\begin{aligned}
& u_{1}=-i R_{0} \Phi_{0} Z_{0} T_{0}\left(1+\frac{m \Lambda^{2}}{E}\right) \mathrm{e}^{\frac{1}{\hbar}\left[i\left(2 E+2 m \varphi-m \Lambda^{2}\right) t+i \frac{\rho}{\Lambda}(E+m \varphi) \phi+\frac{E+m \varphi}{2 \Lambda \rho}\left(\rho^{2}-\rho_{0}^{2}\right)\right]} \\
& u_{2}=-i R_{0} \Phi_{0} Z_{0} T_{0}\left(1+\frac{m \Lambda^{2}}{E}\right) \mathrm{e}^{\frac{1}{\hbar}\left[i\left(2 E+2 m \varphi-m \Lambda^{2}\right) t-i \frac{\rho}{\Lambda}(E+m \varphi) \phi+\frac{E+m \varphi}{2 \Lambda \rho}\left(\rho^{2}-\rho_{0}^{2}\right)\right]} \\
& u_{3}=R_{0} \Phi_{0} Z_{0} T_{0} \mathrm{e}^{\frac{1}{\hbar}\left[i\left(2 E+2 m \varphi-m \Lambda^{2}\right) t-i \frac{\rho}{\Lambda}(E+m \varphi) \phi+\frac{E+m \varphi}{2 \Lambda \rho}\left(\rho^{2}-\rho_{0}^{2}\right)\right]} \\
& u_{4}=R_{0} \Phi_{0} Z_{0} T_{0} \mathrm{e}^{\frac{1}{\hbar}\left[i\left(2 E+2 m \varphi-m \Lambda^{2}\right) t+i \frac{\rho}{\Lambda}(E+m \varphi) \phi+\frac{E+m \varphi}{2 \Lambda \rho}\left(\rho^{2}-\rho_{0}^{2}\right)\right]}
\end{aligned}
$$

Let us differentiate short periods of time $t$ from the larger ones, denoted as $\tau$. The set of equations for long trips is, after assuming a constant radius, angle, and speed of light,

$$
\begin{aligned}
& \frac{\partial u_{1}}{\Lambda \partial \tau}=\frac{i}{\hbar \Lambda}\left[E+m\left(-\Lambda^{2}+\varphi\right)\right] u_{1}-\frac{\partial u_{3}}{c \partial \tau} \\
& \frac{\partial u_{2}}{\Lambda \partial \tau}=\frac{i}{\hbar \Lambda}\left[E+m\left(-\Lambda^{2}+\varphi\right)\right] u_{2}+\frac{\partial u_{4}}{c \partial \tau} \\
& \frac{\partial u_{3}}{\Lambda \partial \tau}=\frac{i}{\hbar \Lambda}\left[E+m\left(\Lambda^{2}+\varphi\right)\right] u_{3}-\frac{\partial u_{1}}{c \partial \tau} \\
& \frac{\partial u_{4}}{\Lambda \partial \tau}=\frac{i}{\hbar \Lambda}\left[E+m\left(\Lambda^{2}+\varphi\right)\right] u_{4}+\frac{\partial u_{2}}{c \partial \tau}
\end{aligned}
$$

The solution to the differential equation for $u_{3}$ is

$$
E+m k \varphi=\left(E_{\text {star }}+m \varphi\right)\left(\frac{\tau_{0}}{\tau}\right)^{\frac{c}{c+\Lambda}},
$$

where $E_{\text {star }}$ is the energy of the photons emitted by a star. Equation (12) forbids the assumption of null $\tau_{0}$. A tentative idea is to identify $\tau_{0}$ with the origin time of this universe, as is done now in Astronomy. This requires all atoms of closer or farther galaxies to be born at the same time and to emit light depending on their history. Let us try to obtain $\tau_{0}$ from a physical constraint. The solution of Set (11) outputs differential equations with real and imaginary parts. A ground state can be defined as being common to all radiation in at least a subsection of the, for example, visible spectrum. This definition can be made by assuming that the energy changes at the same rate on both real and imaginary parts. In that ground state, 


$$
\tau_{0}=\frac{\hbar}{m \Lambda c}
$$

It is known from quantum mechanics (QM) that Planck's constant cannot be zero. Now, QM imposes another requirement: the photon mass also cannot be zero. Otherwise, photons, as particles, must disintegrate at the instant of creation. Inserting the time $\tau=\tau_{0}+t_{T}$, where $t_{T}$ is the traveling time $c^{-1} d$, the photon energy

$E=h \Lambda^{2} c^{-1} \lambda^{-1}$, and the definition of red-shifting $z=\left(\lambda-\lambda_{\text {star }}\right) / \lambda_{\text {star }}$ into Equation (12) yields,

$$
Z=\left(1+\frac{m \Lambda}{\hbar} d\right)^{\frac{c}{c+\Lambda}}-1 .
$$

Precise values for $z$ ranging from zero to one, show little deviation from the linearity between $z$ and the distance $d$. This deviation completely matches a square root as in Equation (13) if $c \approx \Lambda$. See Figure 1. The idea of an expanding, accelerating, or decelerating universe now becomes unnecessary. On the condition of $\left(m \Lambda d \hbar^{-1}\right) \ll 1$,

$$
z \approx \frac{m \Lambda c}{(c+\Lambda) \hbar} d
$$

and by comparison with the experimental result $z=c^{-1} H_{0} d$, the Hubble's constant $H_{0}$, is:

$$
H_{0} \equiv \frac{m \Lambda c^{2}}{(c+\Lambda) \hbar}
$$

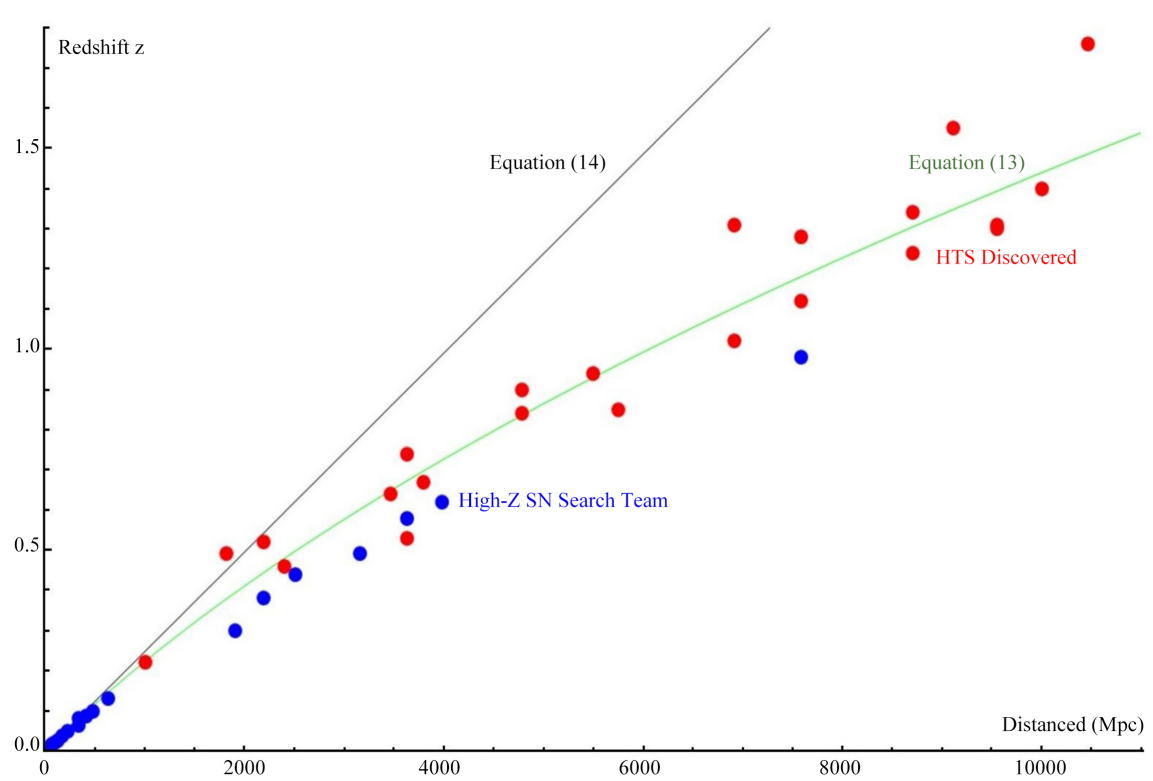

Figure 1. Points coordinates extracted by software from [11], blue data from his Figure (5) and red data from his Figure (6). Green Equation (13) and black Equation (14) were calculated by using $5.643 \times 10^{-69} \mathrm{~kg}$ photon mass. That value is associates with the $74.2 \mathrm{~km}$ $\mathrm{s}^{-1} \mathrm{Mpc}^{-1}$ Hubble's constant reported in [12] according to Equation (15). Note: the green mark was not created by using a fitting function. 


\section{Discussion}

None of the mathematical derivations carried out above will make any sense if the values of the photon mass and speed of light contradict all the experimental work pointing to the specific conditions of a null rest mass and constant speed of light. This paradigm can be solved if the numbers arising from this idea stay inside the corresponding experimental errors.

Regardless, in normal math, if we know the values of the photon mass and the speed limit $\Lambda$, it could be possible to calculate the Hubble's constant. This is not possible now because according to the actual understanding of physics, the photon mass has a null value. Another idea is to work in the opposite direction and estimate the photon mass to confirm whether the model introduced here makes any sense.

With $H_{0}=74.2 \mathrm{~km} \cdot \mathrm{s}^{-1} \cdot \mathrm{Mpc}^{-1}[12]$, by making $\Lambda \approx c \approx 2.9979 \times 10^{5} \mathrm{~km} \cdot \mathrm{s}^{-1}$ and $\hbar=1.0546 \times 10^{-34} \mathrm{~J} \cdot \mathrm{s}$, Equation (15) output a $5.643 \times 10^{-69} \mathrm{~kg}$ photon mass. That photon mass is so small that they can be ignored in any non-astronomical theoretical or experimental work.

Now, we can check the last pending criterion, which is the numerical relationship between the speed of light and the speed limit. Combining the photon energy $E=m \Lambda^{2}\left(1-\beta^{2}\right)^{-1 / 2}$ with the relativistic energy $E^{2}=m^{2} \Lambda^{4}+p^{2} \Lambda^{2}$ results in a function that depends on the repetition-length:

$$
\frac{c}{\Lambda} \approx 1-\frac{1}{2}\left(\frac{m \lambda \Lambda}{h}\right)^{2} .
$$

For example, with yellow, $\lambda=580 \mathrm{~nm}, \beta=c \Lambda^{-1}=1-1.1 \times 10^{-66}$. The last value correlate well with the idea of a constant speed of light as a practical limit velocity for matter.

\section{Conclusions}

Dirac's equation does not change in any way when replacing the electrical charge with another invariant particle scalar and replacing the electrical potentials with the new corresponding potentials. Dirac's equation for gravitational interactions between particles with rest mass automatically outputs some properties for those particles. Some of these properties are two states of matter (called matter and antimatter), spin one (not shown here for lack of space), clockwise counterclockwise rotations, interactions in two orthogonal directions, periodical repetitions in space or time, etc. All these properties match well those of photons if a small sacrifice in theory is made. That sacrifice assumes that photons move at speeds extremely close to the universal speed limit. All the content mentioned in this paragraph could be regarded as a fine theoretical work with no important new consequences for light in relatively small spaces.

Another situation involves the large spaces between galaxies. The energy of coherent light loses its invariant condition and decreases as light travels. The redshift arising from that decrease in energy and its variation with distance 
completely match those observed. This is a huge breakthrough that diminishes the cost of accepting that the photon mass and speed are not the accepted values in academia but very close to them.

Any contradiction with other relativistic consequences should not be expected because the deduction performed here originates from an invariant formalism. Successful study of gravitational interactions from a quantum point of view is an important consequence of the model introduced in this paper.

\section{Conflicts of Interest}

The author declares no conflicts of interest regarding the publication of this paper.

\section{References}

[1] Hubble, E. (1925) Cepheids in Spiral Nebulae, American Astronomical Society. 33, 252-255.

[2] Doppler, C. (1846) Beitrage zurfixsternenkunde, 69. Prague. G. Haase Söhne. https://ui.adsabs.harvard.edu

[3] Friedman, A.A. (1922) Über die Krümmung des Raumes. Zeitschrift für Physik, 10, 377-386. https://doi.org/10.1007/BF01332580

[4] Einstein, A. (1905) On the Electrodynamics of Moving Bodies. http://www.fourmilab.ch/etexts/einstein/specrel/www/

[5] Penzias, A.A. and Wilson, R.W. (1965) A Measurement of Excess Antenna Temperature at $4080 \mathrm{Mc} / \mathrm{s}$. Astrophysical Journal, 142, 419-421. https://doi.org/10.1086/148307

[6] Parra, J.L. (2018) Corpuscular Point of View to Explain Light's Properties. Optics and Photonics Journal, 8, 135-145. https://doi.org/10.4236/opj.2018.85013

[7] Dirac, P. (1928) The Quantum Theory of the Electron. Proceedings of the Royal Society A. Proceedings of the Royal Society of London. Series A, 117, 610-624. https://doi.org/10.1098/rspa.1928.0023

[8] Bethe, H. and Salpeter, E. (1957) Quantum Mechanics of One- and Two-Electron Atoms. Academic Press Inc., New York, 63-71. https://doi.org/10.1007/978-3-662-12869-5

[9] Bose, S.N. (1924) Planck's Law and Light Quantum Hypothesis. Zeitschrift für Physik, 26, 178. http://adsabs.harvard.edu/pdf/1994JApA

[10] Planck, M. (1901) On the Law of Distribution of Energy in the Normal Spectrum. Annalen der Physics, 4, 553.

[11] Riess, A.G. (2012) My Path to the Accelerating Universe. Reviews of Modern Physics, 84, 1165-1175. https://doi.org/10.1103/RevModPhys.84.1165

[12] Shajib, A. et al. (2020) STRIDES: A 3.9 Per Cent Measurement of the Hubble Constant from the Strong Lens System Des J0408-5354. Monthly Notices of the Royal Astronomical Society, 494, 6072-6102. https://academic.oup.com/mnras/article/494/4/6072/5813265 


\section{Appendix}

Matrixes used to derive Equation (3):

$$
\begin{array}{ll}
\alpha_{1}=\left[\begin{array}{llll}
0 & 0 & 0 & 1 \\
0 & 0 & 1 & 0 \\
0 & 1 & 0 & 0 \\
1 & 0 & 0 & 0
\end{array}\right], & \alpha_{2}=\left[\begin{array}{cccc}
0 & 0 & 0 & -i \\
0 & 0 & i & 0 \\
0 & -i & 0 & 0 \\
i & 0 & 0 & 0
\end{array}\right] \\
\alpha_{3} & =\left[\begin{array}{cccc}
0 & 0 & 1 & 0 \\
0 & 0 & 0 & -1 \\
1 & 0 & 0 & 0 \\
0 & -1 & 0 & 0
\end{array}\right], \\
\beta & =\left[\begin{array}{cccc}
1 & 0 & 0 & 0 \\
0 & 1 & 0 & 0 \\
0 & 0 & -1 & 0 \\
0 & 0 & 0 & -1
\end{array}\right]
\end{array}
$$

